\title{
Constraints on the trilinear Higgs self coupling from precision observables
}

\section{G. Degrassi, ${ }^{a}$ M. Fedele ${ }^{b}$ and P.P. Giardino ${ }^{c}$}

\author{
${ }^{a}$ Dipartimento di Matematica e Fisica, Università di Roma Tre and \\ INFN - Sezione di Roma Tre, \\ I-00146 Rome, Italy \\ ${ }^{b}$ Dipartimento di Fisica, Università di Roma "La Sapienza" and \\ INFN - Sezione di Roma, \\ I-00185 Rome, Italy \\ ${ }^{c}$ Physics Department, Brookhaven National Laboratory, \\ Upton, New York 11973, U.S.A. \\ E-mail: degrassi@fis.uniroma3.it, marco.fedele@uniroma1.it, \\ pgiardino@bnl.gov
}

ABSTRACT: We present the constraints on the trilinear Higgs self coupling that arise from loop effects in the $W$ boson mass and the effective sine predictions. We compute the contributions to these precision observables of two-loop diagrams featuring an anomalous trilinear Higgs self coupling. We explicitly show that the same anomalous contributions are found if the analysis of $m_{W}$ and $\sin ^{2} \theta_{\text {eff }}^{\text {lep }}$ is performed in a theory in which the scalar potential in the Standard Model Lagrangian is modified by an (in)finite tower of $\left(\Phi^{\dagger} \Phi\right)^{n}$ terms with $\Phi$ the Higgs doublet. We find that the bounds on the trilinear Higgs self coupling from precision observables are competitive with those coming from Higgs pair production.

Keywords: Beyond Standard Model, Higgs Physics

ARXIV EPRINT: 1702.01737 


\section{Contents}

1 Introduction 1

$2 \quad \lambda_{3}$-dependent contributions in $m_{W}$ and $\sin ^{2} \theta_{\text {eff }}^{\text {lep }} \quad 3$

3 Equivalence with a $\left(\Phi^{\dagger} \Phi\right)^{n}$ theory $\quad 5$

4 Results $\quad 8$

5 Conclusions $\quad 10$

$\begin{array}{lll}\text { A Anomalous contributions in } \Delta \hat{r}_{W}^{(2)} \text { and } Y_{\overline{M S}}^{(2)} & 11\end{array}$

\section{Introduction}

The discovery of a new scalar resonance with a mass around $125 \mathrm{GeV}$ at the Large Hadron Collider (LHC) $[1,2]$ opened a new era in high-energy particle physics. The study of the properties of this particle provides strong evidence that it is the Higgs boson of the Standard Model (SM), i.e., a scalar CP-even state whose coupling to the other known particles has a SM-like structure and a strength proportional to their masses [3-5]. At present, the combined analysis based on 7 and $8 \mathrm{TeV}$ LHC data sets [5] shows that the couplings with the vector bosons are found to be compatible with those expected from the SM within a $\sim 10 \%$ uncertainty, while in the case of the heaviest SM fermions (the top, the bottom quarks and the $\tau$ lepton) the compatibility is achieved with an uncertainty of $\sim 15-20 \%$. Concerning the future, the best present estimates $[6,7]$ indicate that at the end of the LHC Run-2 at $\sqrt{s}=13-14 \mathrm{TeV}$ center-of-mass-energy, the fit of the Higgs boson couplings to the vector bosons is expected to reach a $\sim 5 \%$ precision with $300 \mathrm{fb}^{-1}$ luminosity, while the corresponding ones for the fermions, with the exception of the $\mu$ lepton, can reach $\sim 10-15 \%$ precision. Similar estimates for the end of the High Luminosity option indicate a reduction of these numbers by a factor $\sim 2$.

The study of the Higgs self interactions, coming from the scalar potential part in the Standard Model (SM) Lagrangian, is in a completely different status. In the SM, the Higgs potential in the unitary gauge reads

$$
V\left(\phi_{1}\right)=\frac{m_{H}^{2}}{2} \phi_{1}^{2}+\lambda_{3} v \phi_{1}^{3}+\frac{\lambda_{4}}{4} \phi_{1}^{4}
$$

where the Higgs mass $\left(m_{H}\right)$ and the trilinear $\left(\lambda_{3}\right)$ and quartic $\left(\lambda_{4}\right)$ interactions are linked by the relations $\lambda_{4}^{\mathrm{SM}}=\lambda_{3}^{\mathrm{SM}}=\lambda=m_{H}^{2} /\left(2 v^{2}\right)$, where $v=\left(\sqrt{2} G_{\mu}\right)^{-1 / 2}$ is the vacuum expectation value, and $\lambda$ is the coefficient of the $\left(\Phi^{\dagger} \Phi\right)^{2}$ interaction, $\Phi$ being the Higgs doublet field. 
The experimental verification of these relations, that fully characterize the SM as a renormalizable Quantum Field Theory, relies on the measurements of processes featuring at least two Higgs bosons in the final state. However, since the cross sections for this kind of processes are quite small, constraining the Higgs self interaction couplings within few times their predicted SM value is already extremely challenging. In particular, information on $\lambda_{3}$ can be obtained from Higgs pair production with the present bounds on this reaction from $8 \mathrm{TeV}$ data that allow to constrain $\lambda_{3}$ within $\mathcal{O}\left( \pm(15-20) \lambda_{3}^{\mathrm{SM}}\right)$ [8-11]. At $\sqrt{s}=13 \mathrm{TeV}$, the Higgs pair production cross section, in the SM, is around $35 \mathrm{fb}$ in the gluon-fusion channel [12-21] and even smaller in other production mechanisms [22, 23] that suggests, assuming an integrated luminosity of $3000 \mathrm{fb}^{-1}$, that it will be possible to exclude at the LHC only values in the range $\lambda_{3}<-1.3 \lambda_{3}^{\mathrm{SM}}$ and $\lambda_{3}>8.7 \lambda_{3}^{\mathrm{SM}}$ via the $b \bar{b} \gamma \gamma$ signatures [24] or $\lambda_{3}<-4 \lambda_{3}^{\mathrm{SM}}$ and $\lambda_{3}>12 \lambda_{3}^{\mathrm{SM}}$ including also $b \bar{b} \tau \bar{\tau}$ signatures [25]. Concerning the quartic Higgs self-coupling $\lambda_{4}$, its measurement via triple Higgs production seems beyond the reach of the LHC $[26,27]$ due to the smallness of the corresponding cross section (around $0.1 \mathrm{fb}$ ) [20].

In order to constrain the trilinear Higgs self coupling, a complementary strategy based on the precise measurements of single Higgs production and decay processes was recently proposed. In this approach the effects induced at the loop level on single Higgs processes by a modified $\lambda_{3}$ coupling are studied. This approach builds on the assumption that New Physics (NP) couples to the SM via the Higgs potential in such a way that the lowestorder Higgs couplings to the other fields of the SM (and in particular to the top quark and vector bosons) are still given by the SM prescriptions or, equivalently, modifications to these couplings are so small that do not swamp the loop effects one is considering. This strategy was first applied to $Z H$ production at an $e^{+} e^{-}$collider in ref. [28] and later to Higgs production and decay modes at the LHC [29-31].

The aim of this work is twofold. On the one side we apply the same strategy to the precise measurements of the $W$ boson mass, $m_{W}$, and the effective sine, $\sin ^{2} \theta_{\text {eff }}^{\text {lep }}$. In order to constrain $\lambda_{3}$ we look for effects induced by an anomalous Higgs trilinear coupling at the loop level in the predictions of $m_{W}$ and $\sin ^{2} \theta_{\text {eff }}^{\text {lep }}$. Following the approach of ref. [29] we parametrize the effect of NP at the weak scale via a single parameter $\kappa_{\lambda}$, i.e. the rescaling of the SM trilinear coupling $\lambda_{3}^{\mathrm{SM}}$, so that the $\phi_{1}^{3}$ interaction in the potential is given by

$$
V_{\phi_{1}^{3}}=\lambda_{3} v \phi_{1}^{3} \equiv \kappa_{\lambda} \lambda_{3}^{\mathrm{SM}} v \phi_{1}^{3}, \quad \lambda_{3}^{\mathrm{SM}} \equiv \frac{G_{\mu}}{\sqrt{2}} m_{H}^{2},
$$

and compute, in the unitary gauge, the effects induced by $\kappa_{\lambda}$ in the two-loop $W$ and $Z$ boson self-energies, which are the relevant quantities entering in the two-loop determination of $m_{W}$ and $\sin ^{2} \theta_{\text {eff }}^{\text {lep }}$. On the other side we specify better the anomalous coupling approach employed above by showing that, at the order we are working, i.e. at the two-loop level, it is equivalent to the use of a SM Lagrangian with a scalar potential given by an (in)finite tower of $\left(\Phi^{\dagger} \Phi\right)^{n}$ terms. Furthermore, we show that the use of the unitary gauge in the anomalous coupling approach does not introduce any gauge-dependent problematics.

The paper is organised as follows. In section 2 we discuss the contributions induced by an anomalous Higgs trilinear coupling in $m_{W}$ and $\sin ^{2} \theta_{\text {eff }}^{\text {lep }}$ Section 3 is devoted to show that 
the addition to the SM Lagrangian of $\left(\Phi^{\dagger} \Phi\right)^{n}$ terms gives rise to the same contributions. In the following section we discuss the constraints on $\lambda_{3}$ that can be obtained from the current data. In the last section we summarise and draw our conclusions.

\section{$2 \quad \lambda_{3}$-dependent contributions in $m_{W}$ and $\sin ^{2} \theta_{\text {eff }}^{\text {lep }}$}

We consider a Beyond-the-Standard-Model (BSM) scenario, described at low energy by the SM Lagrangian with a modified scalar potential. We further assume that only Higgs self couplings will be affected by this modified potential while the strength of the couplings of the Higgs to fermions and vector bosons will not change with respect to its SM value, or, equivalently, that any modification of these couplings is going to induce effects much smaller than the ones coming from the "deformation" of the Higgs self couplings.

In the $\overline{M S}$ formulation of the radiative corrections [32-34] the theoretical predictions of $m_{W}$ and $\sin ^{2} \theta_{\text {eff }}^{\text {lep }}$ are expressed in terms of the pole mass of the particles, the $\overline{M S}$ Weinberg angle $\hat{\theta}_{W}(\mu)$ and the $\overline{M S}$ electromagnetic coupling $\hat{\alpha}(\mu)$, defined at the 't-Hooft mass scale $\mu$, usually chosen to be equal to $m_{Z}$. In particular, given the radiative parameters $\Delta \hat{r}_{W}$, $\Delta \hat{\alpha}, Y_{\overline{M S}}$ defined through $\left(\sin ^{2} \hat{\theta}_{W}\left(m_{Z}\right) \equiv \hat{s}^{2}\right)[35]$

$$
\begin{aligned}
\frac{G_{\mu}}{\sqrt{2}} & =\frac{\pi \hat{\alpha}\left(m_{Z}\right)}{2 m_{W}^{2} \hat{s}^{2}}\left(1+\Delta \hat{r}_{W}\right), \quad \hat{\alpha}\left(m_{Z}\right)=\frac{\alpha}{1-\Delta \hat{\alpha}\left(m_{Z}\right)}, \\
\hat{\rho} & \equiv \frac{m_{W}^{2}}{m_{Z}^{2} \hat{c}^{2}}=\frac{1}{1-Y_{\overline{M S}}},
\end{aligned}
$$

with $\hat{c}^{2}=1-\hat{s}^{2}, m_{W}$ is obtained from $m_{Z}, \alpha, G_{\mu}$ via

$$
m_{W}^{2}=\frac{\hat{\rho} m_{Z}^{2}}{2}\left\{1+\left[1-\frac{4 \hat{A}^{2}}{m_{Z}^{2} \hat{\rho}}\left(1+\Delta \hat{r}_{W}\right)\right]^{1 / 2}\right\}
$$

where $\hat{A}=\left(\pi \hat{\alpha}\left(m_{Z}\right) /\left(\sqrt{2} G_{\mu}\right)\right)^{1 / 2}$, while the effective sine is related to $\hat{s}^{2}$ via

$$
\sin ^{2} \theta_{\text {eff }}^{\text {lep }}=\hat{k}_{\ell}\left(m_{Z}^{2}\right) \hat{s}^{2}, \quad \hat{k}_{\ell}\left(m_{Z}^{2}\right)=1+\delta \hat{k}_{\ell}\left(m_{Z}^{2}\right),
$$

where $\hat{k}_{\ell}\left(q^{2}\right)$ is an electroweak form factor ${ }^{1}$ (see ref. [36]) and

$$
\hat{s}^{2}=\frac{1}{2}\left\{1-\left[1-\frac{4 \hat{A}^{2}}{m_{Z}^{2} \hat{\rho}}\left(1+\Delta \hat{r}_{W}\right)\right]^{1 / 2}\right\} .
$$

In our BSM scenario the modifications of the scalar potential affect the radiative parameters $\Delta \hat{r}_{W}$ and $Y_{\overline{M S}}$ at the two-loop level while $\Delta \hat{\alpha}$ and $\delta \hat{k}_{\ell}\left(m_{Z}^{2}\right)$ are going to be affected only at three loops. Recalling that the present knowledge of $m_{W}$ and $\sin ^{2} \theta_{\text {eff }}^{\text {lep }}$ in the SM includes the complete two-loop corrections, we are going to discuss only the

\footnotetext{
${ }^{1}$ In our $\overline{M S}$ formulation the top contribution is not decoupled. Then $\hat{k}$ is very close to 1 and $\sin ^{2} \theta_{\text {eff }}^{\text {lep }}$ can be safely identified with $\hat{s}^{2}[36]$.
} 


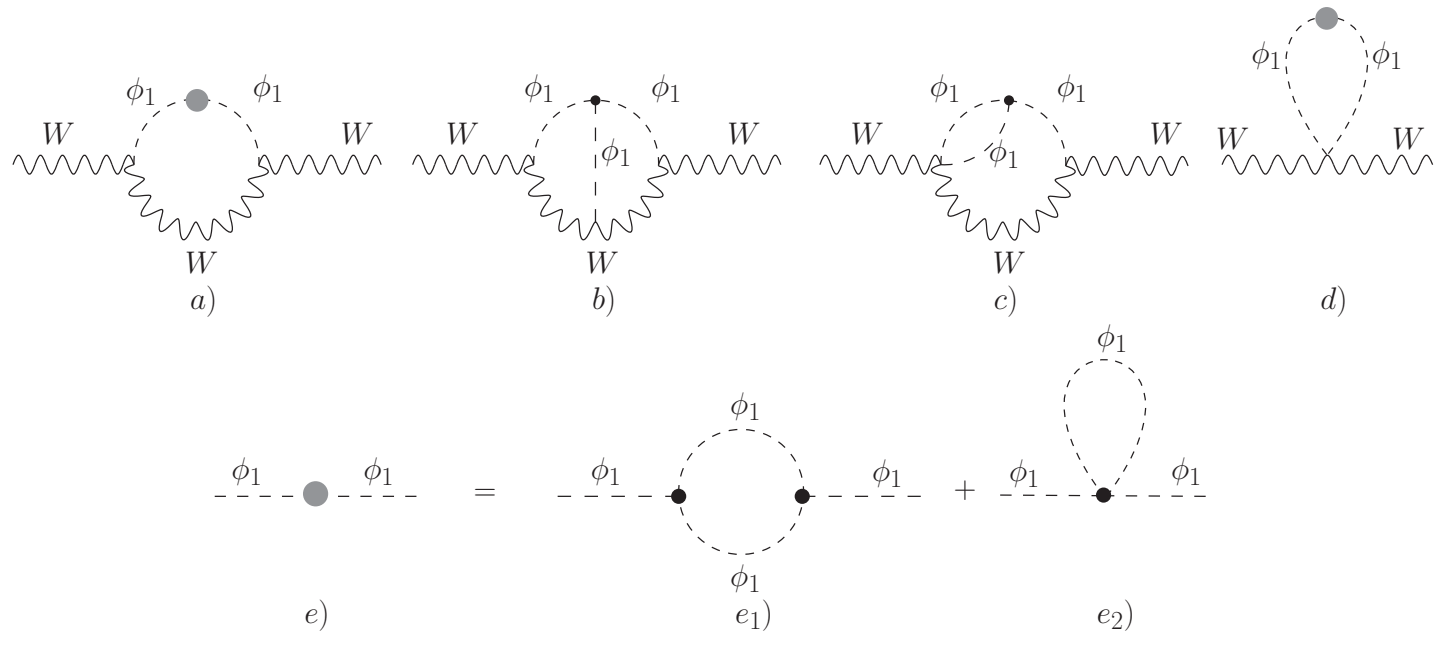

Figure 1. Two-loop $\lambda_{3}$-and- $\lambda_{4}$-dependent diagrams in the $W$ self-energy, in the unitary gauge. The dark blob represent the insertion of the modified diagrams in the one-loop Higgs self energy, shown in the second row. The black point represents either an anomalous $\lambda_{3}$ or $\lambda_{4}$.

modifications induced in $\Delta \hat{r}_{W}$ and $Y_{\overline{M S}}$. The two-loop contribution to $\Delta \hat{r}_{W}$ and $Y_{\overline{M S}}$ can be expressed as [35]

$$
\begin{aligned}
\Delta \hat{r}_{W}^{(2)} & =\frac{\operatorname{Re} A_{W W}^{(2)}\left(m_{W}^{2}\right)}{m_{W}^{2}}-\frac{A_{W W}^{(2)}(0)}{m_{W}^{2}}+\ldots \\
Y_{\overline{M S}}^{(2)} & =\operatorname{Re}\left[\frac{A_{W W}^{(2)}\left(m_{W}^{2}\right)}{m_{W}^{2}}-\frac{A_{Z Z}^{(2)}\left(m_{Z}^{2}\right)}{m_{Z}^{2}}\right]+\ldots
\end{aligned}
$$

where $A_{W W}\left(A_{Z Z}\right)$ is the term proportional to the metric tensor in the $W(Z)$ self energy with the superscript indicating the loop order, and the dots represent additional two-loop contributions that are not sensitive to a modification of the scalar potential.

From the knowledge of the additional contributions induced in $\Delta \hat{r}_{W}^{(2)}$ and $Y_{\overline{M S}}^{(2)}$ one can easily obtain the modification of the radiative parameters $\Delta r$ and $\kappa_{e}\left(m_{z}^{2}\right)$ of the On-Shell (OS) scheme [37]. Considering only new contributions from the modified scalar potential one can write

$$
\Delta r^{(2)}=\Delta \hat{r}_{W}^{(2)}-\frac{c^{2}}{s^{2}} Y_{\overline{M S}}^{(2)},
$$

where $c^{2} \equiv m_{W}^{2} / m_{Z}^{2}, s^{2}=1-c^{2}$ with $\Delta r$ being the radiative parameter entering the $m_{W}-m_{Z}$ interdependence. The effective sine is related to $s^{2}$ in the OS scheme via $\sin ^{2} \theta_{\text {eff }}^{\text {lep }}=$ $\kappa_{e}\left(m_{Z}^{2}\right) s^{2}$ and for the new contributions in $\kappa_{e}\left(m_{Z}^{2}\right)$ one can write

$$
\kappa_{e}^{(2)}\left(m_{Z}^{2}\right)=1-\frac{c^{2}}{s^{2}} Y_{\overline{M S}}^{(2)} .
$$

The new contribution in the self energies in eqs. (2.5), (2.6) can be parametrized just by a modification of the trilinear coupling as described in eq. (1.2). In order to correctly identify the effects related to the $\phi_{1}^{3}$ interaction we follow ref. [29] and work in the unitary gauge. Here we discuss the $W$ self energy but an identical analysis can be done also for the $Z$ self energy. 
The two-loop diagrams in the $W$ self energy that are sensitive to a modification of the Higgs self couplings are depicted in figure 1. The dark blob in diagrams 1a), 1d) represents the one-loop Higgs self energy or the one-loop Higgs mass counterterm that in our scenario gets modified with respect to the SM result in the unitary gauge by the diagrams in figure 1e). The amplitudes of the diagrams in figure 1 were generated using the Mathematica package FeynArts [38] and reduced to scalar Master Integrals using private codes and the packages FeynCalc [39, 40] and Tarcer [41]. After the reduction to scalar integrals we were left with the evaluation of two-loop vacuum integrals and two-loop self-energy diagrams at external momenta different from zero. The former integrals were evaluated analytically using the results of ref. [42]. The latter ones were instead reduced to the set of loop-integral basis functions introduced in ref. [43]. For their numerical evaluation we used the C program TSIL [44]. Our results are expressed in terms of the OS Higgs mass that specifies the Higgs mass counterterm.

Few observations are in order: i) the insertion of the "cactus" diagram $e_{2}$ ) in diagrams a) and $d$ ) in figure 1 gives rise to a contribution proportional to the quartic Higgs self couplings on which we did not make any assumption. However, this contribution is exactly cancelled by the corresponding Higgs mass counterterms diagram so that the final result does not depend on $\lambda_{4}$. This finding is general and does not depend on the particular scheme used to define the Higgs mass. Using a different Higgs mass definition, like, e.g., an $\overline{M S}$ Higgs mass, $\hat{m}_{H}$, the expression for the $W$ self-energy acquires an explicit $\lambda_{4}$ dependence. However, this dependence is going to be cancelled by the $\lambda_{4}$ dependence of $\hat{m}_{H}$, when the latter is extracted from a physical quantity like the OS mass. ii) We expect the modified potential to contain Higgs self interactions with a number of $\phi_{1}$ fields larger than 4 (quintic, sextic, etc. interactions). However, none of these interactions is going to contribute to the $W$ self energy at the two-loop level. ${ }^{2}$ Thus the new contributions induced by our BSM scalar potential at the two-loop level are only functions of $\kappa_{\lambda}$. iii) The contribution to the physical observables given by the diagram $1 d$ ) vanishes in the differences of self energies (see eqs. (2.5), (2.6)).

As in the case of single Higgs processes the $\lambda_{3}$-dependent contributions can be divided into a part quadratically dependent on $\lambda_{3}$ and another linearly proportional to $\lambda_{3}$. The former is due to the diagram $1 a$ ) with the insertion of diagram 1 $1 e_{1}$ ) and of its corresponding Higgs mass counterterm. The latter is given by diagrams $1 b), 1 c$ ).

\section{Equivalence with a $\left(\Phi^{\dagger} \Phi\right)^{n}$ theory}

In this section we show that the results presented in section 2, where no specific assumption on the BSM scalar potential was made, can be obtained using a SM Lagrangian with a scalar potential of the form

$$
V^{N P}=\sum_{n=1}^{N} c_{2 n}\left(\Phi^{\dagger} \Phi\right)^{n}, \quad \Phi=\left(\begin{array}{c}
\phi^{+} \\
\frac{1}{\sqrt{2}}\left(v+\phi_{1}+i \phi_{2}\right)
\end{array}\right),
$$

\footnotetext{
${ }^{2} \mathrm{~A}$ quintic self interaction gives rise to a two-loop tadpole. However, tadpole contributions cancel in eqs. (2.5), (2.6).
} 
where $N$ can be a finite integer or infinite, and in the latter case we assume the series to be convergent. This is the only constraint we impose on the $c_{2 n}$ coefficients, in particular we do not assume an effective-field-theory (EFT) scaling on them, i.e. $c_{2 n+2} \sim c_{2 n} / \Lambda^{2}$ with $\Lambda$ the scale of NP. The SM potential is recovered setting $N=2$ in eq. (3.1) with $c_{2}=-m^{2}$ and $c_{4}=\lambda$ where $-m^{2}$ is the Higgs mass term in the SM Lagrangian in the unbroken phase.

Defining $\phi_{2 u}=\phi^{+} \phi^{-}+\frac{1}{2} \phi_{2}^{2}$ the $n$-th term in the series can be written as

$$
\left(\Phi^{\dagger} \Phi\right)^{n}=\sum_{k=0}^{n} \sum_{j=0}^{k} \sum_{h=0}^{j}\left(\begin{array}{l}
n \\
k
\end{array}\right)\left(\begin{array}{l}
k \\
j
\end{array}\right)\left(\begin{array}{l}
j \\
h
\end{array}\right) \phi_{2 u}^{n-k}\left(\frac{v^{2}}{2}\right)^{k-j}\left(\frac{\phi_{1}^{2}}{2}\right)^{j-h}\left(v \phi_{1}\right)^{h},
$$

with

$$
\left(\begin{array}{l}
n \\
k
\end{array}\right)\left(\begin{array}{l}
k \\
j
\end{array}\right)\left(\begin{array}{l}
j \\
h
\end{array}\right)=\frac{n !}{(n-k) !(k-j) !(j-h) ! h !},
$$

and its contribution to any Higgs self interaction can be labelled by the triplet $\{k, j, h\}$. For example, the minimum of the potential can be obtained from the triplet $\{n, 1,1\}$ :

$$
\left.\frac{d V^{N P}}{d \phi_{1}}\right|_{\phi_{1}=0}=v \sum_{n=1}^{N} c_{2 n} n\left(\frac{v^{2}}{2}\right)^{n-1}=0,
$$

while the Higgs mass is given by the two triplets $\{n, 1,0\}$ and $\{n, 2,2\}$. However, due to the condition in eq. (3.4), the first one is giving a vanishing contribution so that

$$
m_{H}^{2}=v^{2} \sum_{n=1}^{N} c_{2 n} n(n-1)\left(\frac{v^{2}}{2}\right)^{n-2} .
$$

The potential $V^{N P}$ up to quartic interactions can be written as

$$
\begin{aligned}
V_{4 \phi}^{N P}= & \frac{m_{H}^{2}}{2 v^{2}}\left[\phi^{+} \phi^{-}\left(\phi^{+} \phi^{-}+\phi_{2}^{2}\right)+\frac{1}{4} \phi_{2}^{4}\right]+\left(\frac{m_{H}^{2}}{2 v^{2}}+d \lambda_{4}\right) \frac{1}{4} \phi_{1}^{4} \\
& +\left(\frac{m_{H}^{2}}{2 v^{2}}+3 d \lambda_{3}\right) \phi_{1}^{2}\left[\phi^{+} \phi^{-}+\frac{1}{2} \phi_{2}^{2}\right]+\left(\frac{m_{H}^{2}}{2 v}+v d \lambda_{3}\right) \phi_{1}^{3} \\
& +\frac{m_{H}^{2}}{2 v} \phi_{1}\left(\phi_{2}^{2}+2 \phi^{+} \phi^{-}\right)+\frac{1}{2} m_{H}^{2} \phi_{1}^{2} .
\end{aligned}
$$

with

$$
\begin{aligned}
& d \lambda_{3}=\frac{1}{3} \sum_{n=3}^{N} c_{2 n} n(n-1)(n-2)\left(\frac{v^{2}}{2}\right)^{n-2}, \\
& d \lambda_{4}=\frac{2}{3} \sum_{n=3}^{N} c_{2 n} n^{2}(n-1)(n-2)\left(\frac{v^{2}}{2}\right)^{n-2} .
\end{aligned}
$$

It is worth noting that in eq. (3.6) only few couplings are modified with respect to their $\mathrm{SM}$ values. In particular, concerning the unphysical scalars, only the coupling of $\phi_{2 u} \phi_{1}^{2}$ is modified, with a deformation that is related to the deformation of $\lambda_{3}$.

In order to show that the result for the two-loop $W$ self energy computed using $V^{N P}$ is egual to the one obtained assuming an anomalous $\lambda_{3}$ working in the unitary gauge, we have 

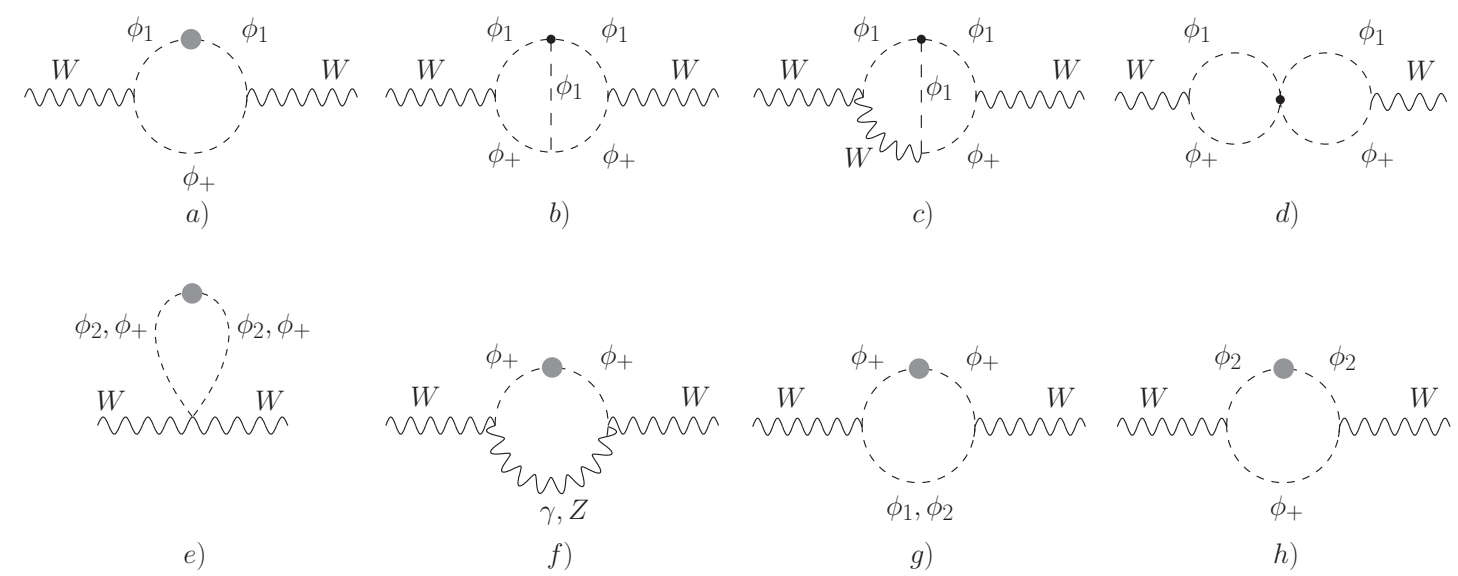

Figure 2. Two-loop diagrams in the $W$ self-energy, involving unphysical scalars where modified couplings (black points) from $V_{4 \phi}^{N P}$ appear. The dark blob represents the insertion of the relevant one-loop self energy (see figure 3 ).

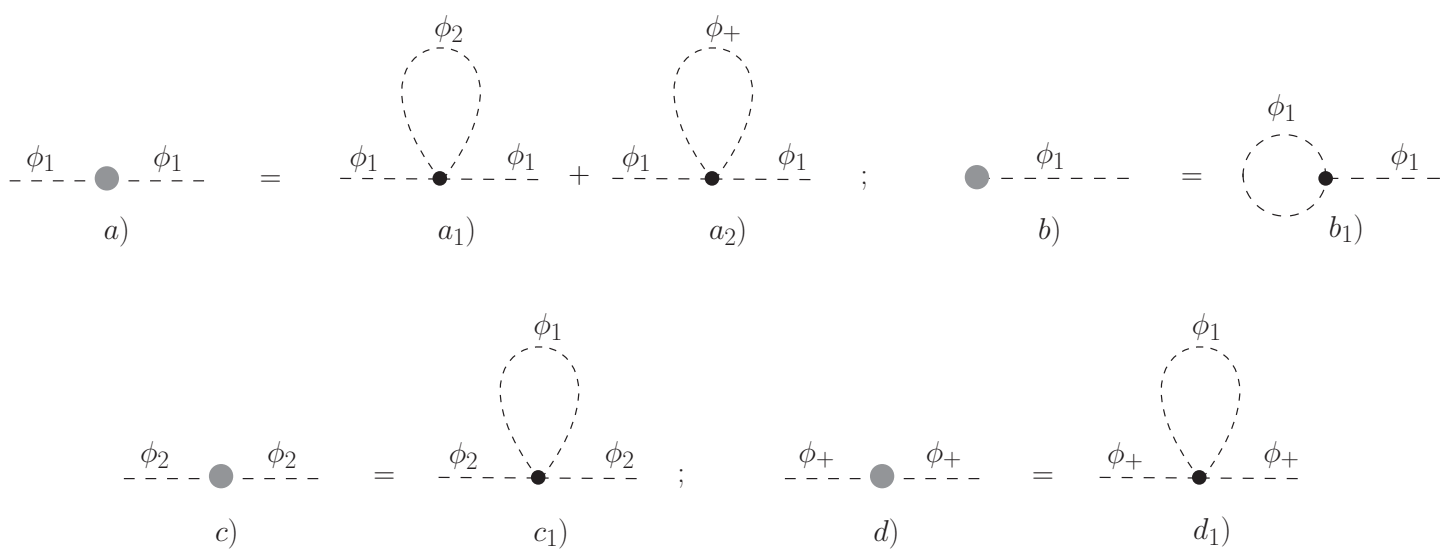

Figure 3. One-loop self energy and tadpole diagrams that contain modified couplings with respect to the SM.

to analyze the two-loop diagrams that are modified with respect to their SM result working in a generic $R_{\xi}$ gauge. Besides the ones in figure 1 , now computed in an $R_{\xi}$ gauge, the diagrams containing unphysical scalars, shown in figure 2 , should be taken into account. In the latter figure the dark blob represents the insertion of the relevant one-loop self energy. In figure 3 we show for the various self energies and the tadpole only the diagrams that are modified with respect to their SM result due to the new scalar potential $V^{N P}$. It easy to show that the only non-vanishing contributions in figures $1 a), 1 d), 2 a$ ) come from the insertion of diagram $e_{1}$ ) in figure 1 plus its corresponding counterterm diagram while all the other insertions being of the cactus type (see $1 e_{2}$ ) and $3 a$ )) are cancelled against the corresponding Higgs mass counterterm diagrams. Furthermore the sum of diagrams 1a) and $2 a$ ) is gauge invariant. Similarly one can prove that the sum of diagrams $1 b), 1 c), 2 b$ ), and $2 c$ ), is gauge invariant.

To complete our proof about the equivalence of the two computations we have to show that the additional contributions with respect to the SM results in the diagrams $2 d)-2 h$ ) 


\begin{tabular}{|c|r|r|}
\hline & $C_{1}$ & \multicolumn{1}{c|}{$C_{2}$} \\
\hline$m_{W}$ & $6.27 \times 10^{-6}$ & $-1.72 \times 10^{-6}$ \\
$\sin ^{2} \theta_{\text {eff }}^{\text {lep }}$ & $-1.56 \times 10^{-5}$ & $4.55 \times 10^{-6}$ \\
\hline
\end{tabular}

Table 1. Values of the coefficients $C_{1}$ and $C_{2}$.

and in the corresponding counterterm diagrams must vanish. Diagram $2 d$ ) is automatically zero, while in the remaining diagrams a self energy of an unphysical scalar is always present. According to $V_{4 \phi}^{N P}$ the only modified contributions in the one-loop self energies of the unphysical scalars are given by diagrams $3 c_{1}$ ) and $3 d_{1}$ ). To the contribution of diagrams $2 e)-2 h$ ) with the insertion of $3 c_{1}$ ) or $3 d_{1}$ ) one has to add the counterterm diagrams. The counterterm associated to the renormalization of the mass of an unphysical scalar contains a term related to the mass of the corresponding vector boson plus a term that is related to the renormalization of the vacuum. The former is not affected by our modified scalar potential. The latter, when $v$ is identified with the minimum of the radiatively corrected potential, is given by the tadpole contribution [45]. Then the only modified contribution in the mass renormalization of the unphysical scalars is given by diagram $3 b_{1}$ ). Thus, the additional contributions with respect to the SM result in the diagrams $2 e)-2 h$ ) are exactly cancelled by the additional contributions in the unphysical scalar mass counterterm diagrams. The key point in this cancellation is the fact that the modification in the vertex with three physical Higgses and the one in the vertices containing two physical and two unphysical Higgses are related by a factor $3 / v$ as shown in eq. (3.6).

We have shown that in a theory with a scalar potential given by eq. (3.1) the twoloop $W$ self energy is modified with respect to its SM value by additional contributions that are gauge-invariant. Then, one can directly compute them in the unitary gauge, that corresponds to the computation with an anomalous $\lambda_{3}$ once the identification $\kappa_{\lambda}=$ $1+2 v^{2} / m_{H}^{2} d \lambda_{3}$ is made.

\section{Results}

The analytic expressions for the contributions induced in $\Delta \hat{r}_{W}^{(2)}$ and $Y_{\overline{M S}}^{(2)}$ by an anomalous $\lambda_{3}$ are reported in the appendix. These contributions are going to modify the SM

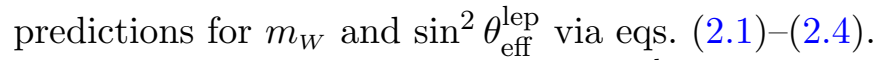

Denoting as $O$ either $m_{W}$ or $\sin ^{2} \theta_{\text {eff }}^{\text {lep }}$ one can write

$$
O=O^{\mathrm{SM}}\left[1+\left(\kappa_{\lambda}-1\right) C_{1}+\left(\kappa_{\lambda}^{2}-1\right) C_{2}\right]
$$

with the values of the coefficients $C_{1}$ and $C_{2}$ reported in table 1 .

Let us comment on the validity of eq. (4.1). At the two-loop level we are working, the contributions induced by an anomalous Higgs trilinear coupling in the precision observables are finite (see table 1 or the appendix), i.e. they are not sensitive to the NP scale $\Lambda$ associated with the modification of the potential. This situation is analogous to what happens in single Higgs processes where new contributions induced by an anomalous $\lambda_{3}$ at 

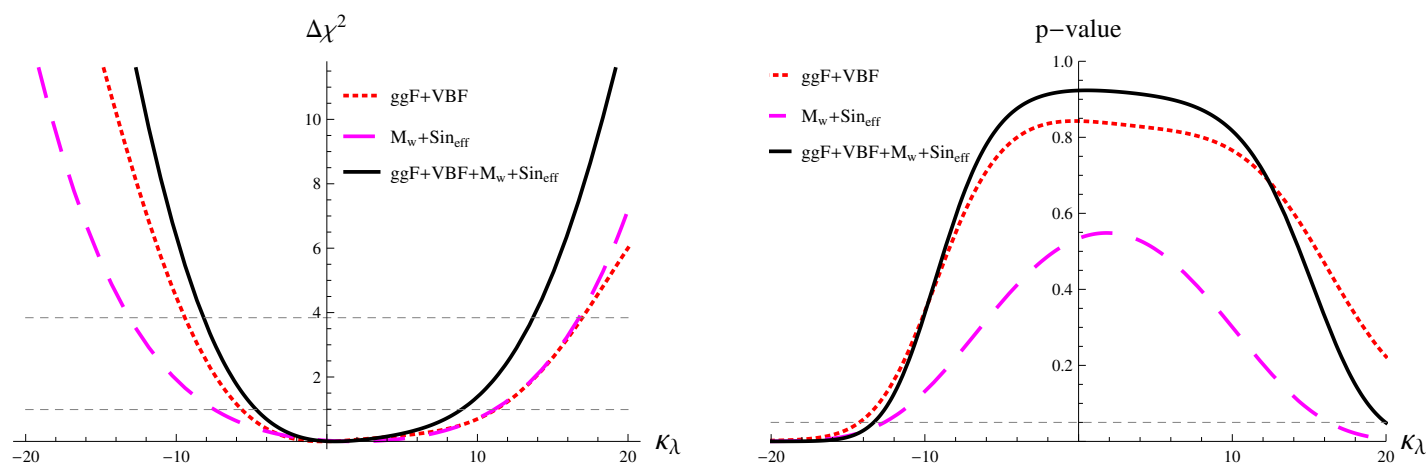

Figure 4. Left: $\chi^{2}$ for the different sets of observables described in the text, the two horizontal lines represent $\Delta \chi^{2}=1$ and $\Delta \chi^{2}=3.84$. Right: corresponding $p$-value, the horizontal line is $p=0.05$.

the NLO are also finite [29]. As in single Higgs processes if NNLO effects are considered, one expects that at three or more loops the modified potential is going to induce contributions not only proportional to $\lambda_{3}$ but also to quartic, quintic etc. Higgs self interactions and moreover these contributions will be sensitive to the NP scale.

The constraints on $\kappa_{\lambda}$ we are going to derive below assume the validity of a perturbative approach. Then, we expect any higher-order contribution to be subdominant with respect to the effects we are computing. This implies that these higher-order contributions should not contain any large amplifying factor related to the scale $\Lambda$, or equivalently that $\Lambda$ cannot be too far from the Electroweak scale. Furthermore, since at the three-loop level one expects the anomalous contribution from the trilinear coupling to grow as $\kappa_{\lambda}^{4}$, a restricted range of $\kappa_{\lambda}$ should also be imposed. Following ref. [29] we consider $\left|\kappa_{\lambda}\right| \lesssim 20$ as a range of validity of our perturbative approach.

In order to set limits on $\kappa_{\lambda}$ from the analysis of precision observables, we perform a simplified fit. We define the best value of $\kappa_{\lambda}$ as the one that minimizes the $\chi^{2}\left(\kappa_{\lambda}\right)$ function defined as

$$
\chi^{2}\left(\kappa_{\lambda}\right) \equiv \sum \frac{\left(O_{\exp }-O_{\text {the }}\right)^{2}}{(\delta)^{2}},
$$

where $O_{\exp }$ refers to the experimental measurement of the observable $O, O_{\text {the }}$ is its theoretical value obtained from eq. (4.1) and $\delta$ is the total uncertainty, that we take as the sum in quadrature of the experimental and theory errors. In order to ascertain the goodness of our fit, we also compute the $p$-value as a function of $\kappa_{\lambda}$ :

$$
p \text {-value }\left(\kappa_{\lambda}\right)=1-F_{\chi_{(n)}^{2}}\left(\chi^{2}\left(\kappa_{\lambda}\right)\right)
$$

where $F_{\chi_{(n)}^{2}}\left(\chi^{2}\left(\kappa_{\lambda}\right)\right)$ is the cumulative distribution function for a $\chi^{2}$ distribution with $n$ degrees of freedom, computed at $\chi^{2}\left(\kappa_{\lambda}\right)$.

In the fit we consider not only the two precision observables but also the signal strength parameter for single Higgs production in gluon fusion $(g g \mathrm{~F})$ and vector boson fusion (VBF). The latter observables were indicated as the $P_{2}$ set in ref. [29] where it was shown that they were returning the most stringent bound on $\kappa_{\lambda}$. We then considered three set of data: 
- The $P_{2}$ set in ref. [29]. The experimental results are presented in table 8 of ref. [5]. See ref. [29] for more details.

- The $\mathrm{W}$ mass and effective sine. For the $\mathrm{W}$ mass we use the latest result by the ATLAS collaboration $m_{W}=80.370 \pm 0.019 \mathrm{GeV}$ [46]. This number, although it has a slightly larger uncertainty with respect to the world average $m_{W}=80.385 \pm 0.015 \mathrm{GeV}$ [47], it is closer to the SM prediction $m_{W}=80.357 \pm 0.009 \pm 0.003$ where the errors refer to the parametric and theoretical uncertainties [35]. Concerning the effective sine, we use the average of the CDF [48] and D0 [49] combinations $\sin ^{2} \theta_{\text {eff }}^{\text {lep }}=0.23185 \pm 0.00035$ [47], to confront against the SM result $\sin ^{2} \theta_{\text {eff }}^{\text {lep }}=0.23145 \pm 0.00012 \pm 0.00005$, where again the errors refer to parametric and theoretical uncertainties respectively [35, 50].

- The combination of these two sets of data.

The $\chi^{2}\left(\kappa_{\lambda}\right)$ and $p$-value functions for the three sets are reported in figure 4 . In particular for the combination we find

$$
\kappa_{\lambda}^{\text {best }}=0.5, \quad \kappa_{\lambda}^{1 \sigma}=[-4.7,8.9], \quad \kappa_{\lambda}^{2 \sigma}=[-8.2,13.7]
$$

where the $\kappa_{\lambda}^{\text {best }}$ is the best value and $\kappa_{\lambda}^{1 \sigma}, \kappa_{\lambda}^{2 \sigma}$ are respectively the $1 \sigma$ and $2 \sigma$ intervals. We identified the $1 \sigma$ and $2 \sigma$ intervals assuming a $\chi^{2}$ distribution. The comparison between the numbers in eq. (4.4) and the corresponding ones for the $g g \mathrm{~F}+\mathrm{VBF}$ case [29], namely

$$
\kappa_{\lambda}^{\text {best }}=-0.24, \quad \kappa_{\lambda}^{1 \sigma}=[-5.6,11.2], \quad \kappa_{\lambda}^{2 \sigma}=[-9.4,17.0] \quad\left(P_{2} \text { set }\right),
$$

shows that the inclusion of the precision observables reduces the allowed range for $\kappa_{\lambda}$. Similarly, looking at the solid black line in the $p$-value part of figure 4 , we can exclude at more than $2 \sigma$ models with $\kappa_{\lambda}$ in the regions $\kappa_{\lambda}<-13.3$ and $\kappa_{\lambda}>20.0$.

These results indicate that in the future, when more accurate measurements will be

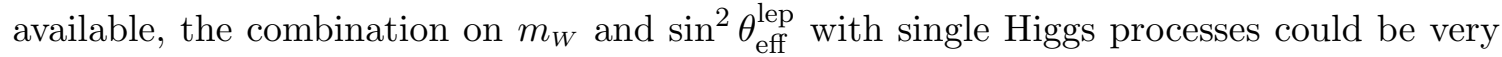
powerful in constraining the allowed region for $\kappa_{\lambda}$, in particular the region of positive $\kappa_{\lambda}$.

\section{Conclusions}

In this work we have discussed how the predictions of the $W$ boson mass and the effective sine are affected by loops featuring an anomalous trilinear Higgs coupling. Following ref. [29] we have chosen to present our results in the contest of the $\kappa$-framework, parametrising the effect of NP at the weak scale via a single parameter, $\kappa_{\lambda}$, i.e. the rescaling of the SM Higgs trilinear coupling. Indeed, given a generic scalar potential constructed using only the Higgs doublet field, at the two-loop level these precision observables are only sensitive to the modification of the trilinear Higgs coupling. As in ref. [29] we worked in the unitary gauge to easily identify the effects we were looking for. We proved that the latter choice is just a technical trick and does not introduce any gauge-dependent issues. In fact, we have explicitly shown that our approach is equivalent, to the order we were working, to an analysis of $m_{W}$ and $\sin ^{2} \theta_{\text {eff }}^{\text {lep }}$ in a generic $R_{\xi}$ gauge performed in a theory described by 
a SM Lagrangian in which the scalar potential is modified by the addition of an (in)finite tower of $\left(\Phi^{\dagger} \Phi\right)^{n}$ terms.

Concerning this scalar potential, one important point to remark is the fact that we did not make any assumption on the size of the coefficients of the various terms in the potential, so that in principle we do not have a priori any restriction on $\kappa_{\lambda}$, apart from the requirement of perturbativity. This is at variance with an EFT approach based on the addition to the SM Lagrangian of a dimension six $\left(\Phi^{\dagger} \Phi\right)^{3}$ term $[30,31]$ where the requirement of $v$ being a global minimum constraints $\kappa_{\lambda}<3$ [51, 52].

However, in order to keep under control higher-order effects induced by quartic, quintic etc. Higgs self interaction some relations among the $c_{2 n}$ coefficients in the potential should be assumed. In particular, either one assumes that $c_{2 n}$ exhibit a scaling with the order $n$ so that the couplings of the interactions with a large number of $\phi_{1}$ do not grow (as an example $d \lambda_{4}$ does not become larger than $d \lambda_{3}$, see eqs. (3.7), (3.8)) or that the various $c_{2 n}$ are related to each other enforcing cancellations among the various terms in the potential.

As we said a theory with a modified trilinear coupling is expected to be valid up to a scale $\Lambda$ that cannot be too far from the Electroweak scale. An estimate of $\Lambda$ can be obtained by looking when perturbative unitarity is lost in processes like e.g. the annihilation of longitudinal vector bosons into $n$ Higgs bosons, $V_{L} V_{L} \rightarrow n \phi_{1}$ [53]. A preliminary study on this subject indicates that $\Lambda \sim 1-3 \mathrm{TeV}$ [54].

We have estimated the sensitivity of $m_{W}$ and $\sin ^{2} \theta_{\text {eff }}^{\text {lep }}$ to an anomalous trilinear coupling via a one-parameter fit. We have also shown that when the analysis of the precision observables is combined with the one from single Higgs inclusive measurements at the LHC $8 \mathrm{TeV}$, a restricted range of allowed $\kappa_{\lambda}$ is found. The range found is actually competitive with the present bounds obtained from the direct searches of Higgs pair production.

\section{Acknowledgments}

Two of us (G.D., P.P.G.) want to thank their collaborators F. Maltoni and D. Pagani together with Xiaoran Zhao for useful discussions and communications. G.D. wants to thank R. Rattazzi for an enlightening "endless" discussion. The work of P.P.G. is supported by the United States Department of Energy under Grant Contracts de-sc0012704.

\section{A Anomalous contributions in $\Delta \hat{r}_{W}^{(2)}$ and $Y_{\overline{M S}}^{(2)}$}

Here we give the analytic expressions for the additional contributions induced in $\Delta \hat{r}_{W}^{(2)}$ and $Y_{\overline{M S}}^{(2)}$ by an anomalous $\lambda_{3}$. In the formulae below

$$
\zeta_{W}=\frac{m_{H}^{2}}{m_{W}^{2}}, \quad \overline{\ln }(x)=\log \left(\frac{x}{\mu}\right),
$$

with $\mu$ the 't-Hooft mass scale. We find for the $\kappa_{\lambda}$ contributions

$$
\begin{aligned}
\Delta \hat{r}_{W}^{\left(2, \kappa_{\lambda}\right)}= & \left(\frac{\hat{\alpha}}{4 \pi s^{2}}\right)^{2}\left\{\left[\frac{1}{64} \zeta_{W}\left(-12 \zeta_{W}^{2}+49 \zeta_{W}+18\right)+\zeta_{W} \frac{4 \zeta_{W}^{2}-7 \zeta_{W}+6}{16\left(\zeta_{W}-1\right)} \overline{\ln }\left(m_{W}^{2}\right)\right.\right. \\
& +\left(\frac{10-13 \zeta_{W}}{16\left(\zeta_{W}-1\right)} \zeta_{W}^{2}+\frac{-2 \zeta_{W}^{4}+9 \zeta_{W}^{3}-46 \zeta_{W}+60}{32\left(\zeta_{W}-1\right)^{2}} \zeta_{W} \overline{\ln }\left(m_{W}^{2}\right)\right) \overline{\ln }\left(m_{H}^{2}\right)
\end{aligned}
$$




$$
\begin{aligned}
& +\frac{2 \zeta_{W}^{4}-9 \zeta_{W}^{3}+46 \zeta_{W}-60}{64\left(\zeta_{W}-1\right)^{2}} \zeta_{W} \overline{\ln }\left(m_{W}^{2}\right)^{2} \\
& +3 \frac{2 \zeta_{W}^{4}-3 \zeta_{W}^{3}-4 \zeta_{W}^{2}+18 \zeta_{W}-20}{64\left(\zeta_{W}-1\right)^{2}} \zeta_{W} \overline{\ln }\left(m_{H}^{2}\right)^{2} \\
& +\left(\frac{1}{8}\left(\zeta_{W}^{2}-3 \zeta_{W}-2\right) \zeta_{W}+\frac{1}{8}\left(\zeta_{W}-2\right) \zeta_{W} \overline{\ln }\left(m_{W}^{2}\right)\right. \\
& \left.-\frac{1}{8}\left(\zeta_{W}-2\right) \zeta_{W}^{2} \overline{\ln }\left(m_{H}^{2}\right)\right) B_{0}\left(m_{W}^{2}, m_{H}^{2}, m_{W}^{2}\right) \\
& +\frac{1}{8}\left(\zeta_{W}-2\right) \zeta_{W} B_{0}\left(m_{W}^{2}, m_{H}^{2}, m_{W}^{2}\right)^{2}+\frac{\zeta_{W}-2}{8 m_{W}^{2}} \zeta_{W} S_{0}\left(m_{W}^{2}, m_{W}^{2}, m_{H}^{2}, m_{H}^{2}\right) \\
& -\frac{1}{2}\left(\zeta_{W}-1\right) \zeta_{W}^{2} T_{0}\left(m_{W}^{2}, m_{H}^{2}, m_{W}^{2}, m_{H}^{2}\right) \\
& +\frac{1}{4}\left(\zeta_{W}-2\right) \zeta_{W} U_{0}\left(m_{W}^{2}, m_{H}^{2}, m_{W}^{2}, m_{H}^{2}, m_{W}^{2}\right) \\
& -\frac{1}{8} \zeta_{W}\left(\zeta_{W}^{2}+\zeta_{W}-6\right) U_{0}\left(m_{W}^{2}, m_{W}^{2}, m_{H}^{2}, m_{H}^{2}, m_{H}^{2}\right) \\
& +\frac{1}{16} m_{H}^{2}\left(\zeta_{W}^{3}-12 \zeta_{W}+24\right) M_{0}\left(m_{W}^{2}, m_{H}^{2}, m_{H}^{2}, m_{W}^{2}, m_{W}^{2}, m_{H}^{2}\right) \\
& +3 \frac{-2 \zeta_{W}^{3}+\zeta_{W}^{2}+4 \zeta_{W}+24}{64\left(\zeta_{W}-1\right)^{2}} \zeta_{W}^{2} \phi\left(\frac{1}{4}\right)+3 \frac{4 \zeta_{W}^{2}-41 \zeta_{W}+10}{32\left(\zeta_{W}-1\right)^{2}} \zeta_{W} \phi\left(\frac{1}{4 \zeta_{W}}\right) \\
& \left.-\frac{\zeta_{W}\left(2 \zeta_{W}^{4}-13 \zeta_{W}^{3}+18 \zeta_{W}^{2}+40 \zeta_{W}-128\right)}{64\left(\zeta_{W}-1\right)^{2}} \phi\left(\frac{\zeta_{W}}{4}\right)\right] \kappa_{\lambda} \\
& +\left[\frac{\left(-476 \zeta_{W}^{4}+2403 \zeta_{W}^{3}-4995 \zeta_{W}^{2}+1652 \zeta_{W}+120\right)}{256\left(\zeta_{W}-4\right)\left(\zeta_{W}-1\right)} \zeta_{W}\right. \\
& +3 \frac{\left(\zeta_{W}^{4}-6 \zeta_{W}^{3}+39 \zeta_{W}^{2}-100 \zeta_{W}+12\right.}{32\left(\zeta_{W}-4\right)\left(\zeta_{W}-1\right)^{2}} \zeta_{W} \overline{\ln }\left(m_{W}^{2}\right) \\
& +9\left(\frac{5 \zeta_{W}^{4}-31 \zeta_{W}^{3}+80 \zeta_{W}^{2}-84 \zeta_{W}+48}{32\left(\zeta_{W}-4\right)\left(\zeta_{W}-1\right)^{2}} \zeta_{W}^{2}-\frac{27 \zeta_{W}^{2}}{32\left(\zeta_{W}-1\right)^{2}} \overline{\ln }\left(m_{W}^{2}\right)\right) \overline{\ln }\left(m_{H}^{2}\right) \\
& -3 \frac{7 \zeta_{W}^{4}-45 \zeta_{W}^{3}+117 \zeta_{W}^{2}-145 \zeta_{W}+120}{64\left(\zeta_{W}-4\right)\left(\zeta_{W}-1\right)^{2}} \zeta_{W}^{2} \overline{\ln }\left(m_{H}^{2}\right)^{2} \\
& +\left(\frac{1}{32} \zeta_{W}\left(\zeta_{W}^{2}-4 \zeta_{W}+12\right)-\frac{1}{16} \zeta_{W}\left(\zeta_{W}^{2}-4 \zeta_{W}+12\right) \overline{\ln }\left(m_{H}^{2}\right)\right. \\
& \left.-9 \frac{\left(\zeta_{W}-2\right)^{3}}{32\left(\zeta_{W}-4\right)} \zeta_{W} B_{0}\left(m_{H}^{2}, m_{H}^{2}, m_{H}^{2}\right)\right) B_{0}\left(m_{W}^{2}, m_{H}^{2}, m_{W}^{2}\right) \\
& +\left(9 \frac{2 \zeta_{W}^{4}-13 \zeta_{W}^{3}+33 \zeta_{W}^{2}-36 \zeta_{W}+32}{64\left(\zeta_{W}-4\right)\left(\zeta_{W}-1\right)} \zeta_{W}\right. \\
& +9 \frac{\zeta_{W}^{4}-6 \zeta_{W}^{3}+14 \zeta_{W}^{2}-8 \zeta_{W}+8}{32\left(\zeta_{W}-4\right)\left(\zeta_{W}-1\right)^{2}} \zeta_{W} \overline{\ln }\left(m_{W}^{2}\right) \\
& \left.-9 \frac{\zeta_{W}^{4}-7 \zeta_{W}^{3}+19 \zeta_{W}^{2}-24 \zeta_{W}+20}{32\left(\zeta_{W}-4\right)\left(\zeta_{W}-1\right)^{2}} \zeta_{W}^{2} \overline{\ln }\left(m_{H}^{2}\right)\right) B_{0}\left(m_{H}^{2}, m_{H}^{2}, m_{H}^{2}\right) \\
& +9 \frac{\zeta_{W}^{2}-4 \zeta_{W}+8}{32 m_{W}^{2}\left(\zeta_{W}-4\right)} \zeta_{W} S_{0}\left(m_{W}^{2}, m_{W}^{2}, m_{H}^{2}, m_{H}^{2}\right)
\end{aligned}
$$




$$
\begin{aligned}
& -\frac{\zeta_{W}^{3}-5 \zeta_{W}^{2}+16 \zeta_{W}-12}{4\left(\zeta_{W}-4\right)} \zeta_{W} T_{0}\left(m_{W}^{2}, m_{H}^{2}, m_{W}^{2}, m_{H}^{2}\right) \\
& +\frac{7 \zeta_{W}^{3}-38 \zeta_{W}^{2}+52 \zeta_{W}+24}{32\left(\zeta_{W}-4\right)} \zeta_{W} U_{0}\left(m_{W}^{2}, m_{W}^{2}, m_{H}^{2}, m_{H}^{2}, m_{H}^{2}\right) \\
& +3 \frac{7 \zeta_{W}^{4}-45 \zeta_{W}^{3}+99 \zeta_{W}^{2}-64 \zeta_{W}+84}{64\left(\zeta_{W}-4\right)\left(\zeta_{W}-1\right)^{2}} \zeta_{W}^{2} \phi\left(\frac{1}{4}\right) \\
& \left.\left.+27 \frac{4 \zeta_{W}-1}{64\left(\zeta_{W}-1\right)^{2}} \zeta_{W}^{2} \phi\left(\frac{1}{4 \zeta_{W}}\right)\right] \kappa_{\lambda}^{2}\right\} \\
Y_{\overline{M S}}^{\left(2, \kappa_{\lambda}\right)}= & \left(\frac{\hat{\alpha}}{4 \pi s^{2}}\right)^{2}\left\{\left[f_{1}\left(\frac{m_{H}^{2}}{m_{W}^{2}}\right)-\frac{1}{c^{4}} f_{1}\left(\frac{m_{H}^{2}}{m_{Z}^{2}}\right)\right] \kappa_{\lambda}+\left[f_{2}\left(\frac{m_{H}^{2}}{m_{W}^{2}}\right)-\frac{1}{c^{4}} f_{2}\left(\frac{m_{H}^{2}}{m_{Z}^{2}}\right)\right] \kappa_{\lambda}^{2}\right\},
\end{aligned}
$$

where we have defined the functions $f_{1}, f_{2}$ as

$$
\begin{aligned}
& f_{1}\left(\zeta \equiv m_{H}^{2} / m^{2}\right)=\frac{1}{32}\left[-\left(6 \zeta^{2}-11 \zeta-15\right) \zeta+4(2 \zeta-3) \zeta \overline{\ln }\left(m^{2}\right)+(\zeta-4) \zeta^{2} \overline{\ln }\left(m^{2}\right)^{2}\right. \\
& -2\left(10 \zeta^{2}+(\zeta-4) \zeta^{2} \overline{\ln }\left(m^{2}\right)\right) \overline{\ln }\left(m_{H}^{2}\right)+3 \zeta^{3} \overline{\ln }\left(m_{H}^{2}\right)^{2} \\
& +4\left(-2-3 \zeta+\zeta^{2}+(\zeta-2) \overline{\ln }\left(m^{2}\right)\right. \\
& \left.-(\zeta-2) \zeta \overline{\ln }\left(m_{H}^{2}\right)\right) \zeta B_{0}\left(m^{2}, m_{H}^{2}, m^{2}\right) \\
& +4(\zeta-2) \zeta B_{0}\left(m^{2}, m_{H}^{2}, m^{2}\right)^{2}+4(\zeta-2) \frac{\zeta^{2}}{m_{H}^{2}} S_{0}\left(m^{2}, m^{2}, m_{H}^{2}, m_{H}^{2}\right) \\
& -16(\zeta-1) \zeta^{2} T_{0}\left(m^{2}, m_{H}^{2}, m^{2}, m_{H}^{2}\right) \\
& +8(\zeta-2) \zeta U_{0}\left(m^{2}, m_{H}^{2}, m^{2}, m_{H}^{2}, m^{2}\right) \\
& -4\left(\zeta^{2}+\zeta-6\right) \zeta U_{0}\left(m^{2}, m^{2}, m_{H}^{2}, m_{H}^{2}, m_{H}^{2}\right) \\
& +2\left(\zeta^{3}-12 \zeta+24\right) m_{H}^{2} M_{0}\left(m^{2}, m_{H}^{2}, m_{H}^{2}, m^{2}, m^{2}, m_{H}^{2}\right) \\
& \left.-3 \zeta^{3} \phi\left(\frac{1}{4}\right)-(\zeta-4)(\zeta-2) \zeta \phi\left(\frac{\zeta}{4}\right)\right] \text {, } \\
& f_{2}\left(\zeta \equiv m_{H}^{2} / m^{2}\right)=\frac{1}{128}\left[-\frac{\left(238 \zeta^{3}-941 \zeta^{2}+1660 \zeta+60\right) \zeta}{\zeta-4}+\frac{12\left(\zeta^{2}-4 \zeta+12\right) \zeta}{\zeta-4} \overline{\ln }\left(m^{2}\right)\right. \\
& +36 \frac{5 \zeta^{2}-20 \zeta+32}{\zeta-4} \zeta^{2} \overline{\ln }\left(m_{H}^{2}\right)-6 \frac{7 \zeta^{2}-28 \zeta+36}{\zeta-4} \zeta^{2} \overline{\ln }\left(m_{H}^{2}\right)^{2} \\
& +36\left(\frac{\zeta^{3}-5 \zeta^{2}+12 \zeta-16}{\zeta-4} \zeta+\frac{\zeta^{2}-4 \zeta+8}{\zeta-4} \zeta \overline{\ln }\left(m^{2}\right)\right. \\
& \left.-\frac{(\zeta-2)^{2}}{\zeta-4} \zeta^{2} \overline{\ln }\left(m_{H}^{2}\right)-\frac{(\zeta-2)^{3}}{\zeta-4} \zeta B_{0}\left(m^{2}, m_{H}^{2}, m^{2}\right)\right) B_{0}\left(m_{H}^{2}, m_{H}^{2}, m_{H}^{2}\right) \\
& +4\left(\zeta^{2}-4 \zeta+12\right)\left(1-2 \overline{\ln }\left(m_{H}^{2}\right)\right) \zeta B_{0}\left(m^{2}, m_{H}^{2}, m^{2}\right)
\end{aligned}
$$




$$
\begin{aligned}
& +36 \frac{\zeta^{2}-4 \zeta+8}{m_{H}^{2}(\zeta-4)} \zeta^{2} S_{0}\left(m^{2}, m^{2}, m_{H}^{2}, m_{H}^{2}\right) \\
& -32 \frac{\zeta^{3}-5 \zeta^{2}+16 \zeta-12}{\zeta-4} \zeta T_{0}\left(m^{2}, m_{H}^{2}, m^{2}, m_{H}^{2}\right) \\
& +4 \frac{7 \zeta^{3}-38 \zeta^{2}+52 \zeta+24}{\zeta-4} \zeta U_{0}\left(m^{2}, m^{2}, m_{H}^{2}, m_{H}^{2}, m_{H}^{2}\right) \\
& \left.+6 \frac{7 \zeta^{2}-28 \zeta+36}{\zeta-4} \zeta^{2} \phi\left(\frac{1}{4}\right)\right] .
\end{aligned}
$$

In eqs. (A.2)-(A.5)

$$
\phi(x)=4 \sqrt{\frac{x}{1-x}} \operatorname{Im}\left(\operatorname{Li}_{2}\left(e^{i 2 \arcsin (\sqrt{x})}\right)\right),
$$

and, following refs. $[43,44]$, we define the $d$-dimensional functions

$$
\begin{aligned}
B_{0}(s, x, y) & =\lim _{\epsilon \rightarrow 0}\left[B(s, x, y)-\frac{1}{\epsilon}\right]=-\int_{0}^{1} d t \overline{\ln }[t x+(1-t) y-t(1-t) s], \\
S_{0}(s, x, y, z) & =\lim _{\epsilon \rightarrow 0}\left[S(s, x, y, z)+\frac{x+y+z}{2 \epsilon^{2}}\right. \\
& \left.+\frac{\frac{s}{2}-x-y-z}{2 \epsilon}-\frac{A(x)+A(y)+A(z)}{\epsilon}\right], \\
T_{0}(s, x, y, z) & =-\frac{\partial}{\partial x} S_{0}(s, x, y, z), \\
U_{0}(s, x, y, z, u) & =\lim _{\epsilon \rightarrow 0}\left[U(s, x, y, z, u)+\frac{1}{2 \epsilon^{2}}-\frac{1}{2 \epsilon}-\frac{B(s, x, y)}{\epsilon}\right], \\
M_{0}(s, x, y, z, u, v) & =\lim _{\epsilon \rightarrow 0}[M(s, x, y, z, u, v)],
\end{aligned}
$$

with $d=4-2 \epsilon$ and

$$
\begin{aligned}
A(x) & =-i \frac{(2 \pi \mu)^{2 \epsilon}}{\pi^{2}} \int \frac{d^{d} k_{1}}{\left(k_{1}^{2}-x\right)} \\
B(s, x, y) & =-i \frac{(2 \pi \mu)^{2 \epsilon}}{\pi^{2}} \int \frac{d^{d} k_{1}}{\left(k_{1}^{2}-x\right)\left(k_{3}^{2}-y\right)} \\
S(s, x, y, z) & =-\left(\frac{(2 \pi \mu)^{2 \epsilon}}{\pi^{2}}\right)^{2} \iint \frac{d^{d} k_{1} d^{d} k_{2}}{\left(k_{1}^{2}-x\right)\left(k_{5}^{2}-y\right)\left(k_{4}^{2}-z\right)} \\
U(s, x, y, z, u) & =-\left(\frac{(2 \pi \mu)^{2 \epsilon}}{\pi^{2}}\right)^{2} \iint \frac{d^{d} k_{1} d^{d} k_{2}}{\left(k_{5}^{2}-u\right)\left(k_{2}^{2}-x\right)\left(k_{3}^{2}-z\right)\left(k_{4}^{2}-y\right)} \\
M(s, x, y, z, u, v) & =-\left(\frac{(2 \pi \mu)^{2 \epsilon}}{\pi^{2}}\right)^{2} \iint \frac{d^{d} k_{1} d^{d} k_{2}}{\left(k_{1}^{2}-x\right)\left(k_{2}^{2}-y\right)\left(k_{3}^{2}-z\right)\left(k_{4}^{2}-u\right)\left(k_{5}^{2}-v\right)}
\end{aligned}
$$

where we introduced the notation

$$
k_{3}=k_{1}-p, \quad k_{4}=k_{2}-p, \quad k_{5}=k_{1}-k_{2},
$$

with $p^{2}=s$. 
Open Access. This article is distributed under the terms of the Creative Commons Attribution License (CC-BY 4.0), which permits any use, distribution and reproduction in any medium, provided the original author(s) and source are credited.

\section{References}

[1] CMS collaboration, Observation of a new boson at a mass of $125 \mathrm{GeV}$ with the CMS experiment at the LHC, Phys. Lett. B 716 (2012) 30 [arXiv:1207.7235] [INSPIRE].

[2] ATLAS collaboration, Observation of a new particle in the search for the standard model Higgs boson with the ATLAS detector at the LHC, Phys. Lett. B 716 (2012) 1 [arXiv: 1207.7214] [INSPIRE].

[3] CMS collaboration, Precise determination of the mass of the Higgs boson and tests of compatibility of its couplings with the standard model predictions using proton collisions at 7 and 8 TeV, Eur. Phys. J. C 75 (2015) 212 [arXiv: 1412.8662] [InSPIRE].

[4] ATLAS collaboration, Measurements of the Higgs boson production and decay rates and coupling strengths using pp collision data at $\sqrt{s}=7$ and $8 \mathrm{TeV}$ in the ATLAS experiment, Eur. Phys. J. C 76 (2016) 6 [arXiv: 1507.04548] [InSPIRE].

[5] ATLAS and CMS collaboration, Measurements of the Higgs boson production and decay rates and constraints on its couplings from a combined ATLAS and CMS analysis of the LHC pp collision data at $\sqrt{s}=7$ and $8 \mathrm{TeV}$, JHEP 08 (2016) 045 [arXiv:1606.02266] [INSPIRE].

[6] CMS collaboration, Projected performance of an upgraded CMS detector at the LHC and HL-LHC: contribution to the Snowmass process, arXiv: 1307.7135 [INSPIRE].

[7] M.E. Peskin, Estimation of LHC and ILC capabilities for precision Higgs boson coupling measurements, talk given at Community Summer Study 2013: Snowmass on the Mississippi (CSS2013), July 29-August 6, Minneapolis, U.S.A. (2013), arXiv:1312.4974 [INSPIRE].

[8] ATLAS collaboration, Searches for Higgs boson pair production in the $h h \rightarrow b b \tau \tau, \gamma \gamma W W^{*}, \gamma \gamma b b, b b b b$ channels with the ATLAS detector, Phys. Rev. D 92 (2015) 092004 [arXiv: 1509.04670] [INSPIRE].

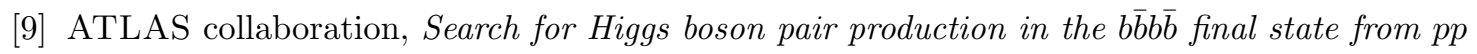
collisions at $\sqrt{s}=8 \mathrm{TeV}$ with the ATLAS detector, Eur. Phys. J. C 75 (2015) 412 [arXiv: 1506.00285] [INSPIRE].

[10] CMS collaboration, Search for two Higgs bosons in final states containing two photons and two bottom quarks in proton-proton collisions at 8 TeV, Phys. Rev. D 94 (2016) 052012 [arXiv: 1603.06896] [INSPIRE].

[11] ATLAS collaboration, Search for pair production of Higgs bosons in the b $\bar{b} b \bar{b}$ final state using proton-proton collisions at $\sqrt{s}=13 \mathrm{TeV}$ with the ATLAS detector, ATLAS-CONF-2016-049 (2016).

[12] E.W.N. Glover and J.J. van der Bij, Higgs boson pair production via gluon fusion, Nucl. Phys. B 309 (1988) 282 [INSPIRE].

[13] T. Plehn, M. Spira and P.M. Zerwas, Pair production of neutral Higgs particles in gluon-gluon collisions, Nucl. Phys. B 479 (1996) 46 [Erratum ibid. B 531 (1998) 655] [hep-ph/9603205] [INSPIRE]. 
[14] S. Dawson, S. Dittmaier and M. Spira, Neutral Higgs boson pair production at hadron colliders: QCD corrections, Phys. Rev. D 58 (1998) 115012 [hep-ph/9805244] [INSPIRE].

[15] J. Grigo, J. Hoff, K. Melnikov and M. Steinhauser, On the Higgs boson pair production at the LHC, Nucl. Phys. B 875 (2013) 1 [arXiv:1305.7340] [INSPIRE].

[16] J. Grigo, K. Melnikov and M. Steinhauser, Virtual corrections to Higgs boson pair production in the large top quark mass limit, Nucl. Phys. B 888 (2014) 17 [arXiv:1408.2422] [INSPIRE].

[17] J. Grigo, J. Hoff and M. Steinhauser, Higgs boson pair production: top quark mass effects at NLO and NNLO, Nucl. Phys. B 900 (2015) 412 [arXiv:1508.00909] [inSPIRE].

[18] G. Degrassi, P.P. Giardino and R. Gröber, On the two-loop virtual QCD corrections to Higgs boson pair production in the Standard Model, Eur. Phys. J. C 76 (2016) 411 [arXiv: 1603.00385] [INSPIRE].

[19] D. de Florian and J. Mazzitelli, Higgs boson pair production at next-to-next-to-leading order in QCD, Phys. Rev. Lett. 111 (2013) 201801 [arXiv:1309.6594] [INSPIRE].

[20] F. Maltoni, E. Vryonidou and M. Zaro, Top-quark mass effects in double and triple Higgs production in gluon-gluon fusion at NLO, JHEP 11 (2014) 079 [arXiv:1408.6542] [INSPIRE].

[21] S. Borowka et al., Higgs boson pair production in gluon fusion at next-to-leading order with full top-quark mass dependence, Phys. Rev. Lett. 117 (2016) 012001 [arXiv:1604.06447] [INSPIRE].

[22] J. Baglio et al., The measurement of the Higgs self-coupling at the LHC: theoretical status, JHEP 04 (2013) 151 [arXiv:1212.5581] [INSPIRE].

[23] R. Frederix et al., Higgs pair production at the LHC with NLO and parton-shower effects, Phys. Lett. B 732 (2014) 142 [arXiv:1401.7340] [InSPIRE].

[24] ATLAS collaboration, Prospects for measuring Higgs pair production in the channel $H(\rightarrow \gamma \gamma) H(\rightarrow b \bar{b})$ using the ATLAS detector at the HL-LHC, ATL-PHYS-PUB-2014-019 (2014).

[25] ATLAS collaboration, Higgs pair production in the $H(\rightarrow \tau \tau) H(\rightarrow b \bar{b})$ channel at the high-luminosity LHC, ATL-PHYS-PUB-2015-046 (2015).

[26] T. Plehn and M. Rauch, The quartic Higgs coupling at hadron colliders, Phys. Rev. D 72 (2005) 053008 [hep-ph/0507321] [INSPIRE].

[27] T. Binoth, S. Karg, N. Kauer and R. Ruckl, Multi-Higgs boson production in the Standard Model and beyond, Phys. Rev. D 74 (2006) 113008 [hep-ph/0608057] [INSPIRE].

[28] M. McCullough, An indirect model-dependent probe of the Higgs self-coupling, Phys. Rev. D 90 (2014) 015001 [arXiv: 1312.3322] [INSPIRE].

[29] G. Degrassi, P.P. Giardino, F. Maltoni and D. Pagani, Probing the Higgs self coupling via single Higgs production at the LHC, JHEP 12 (2016) 080 [arXiv:1607.04251] [INSPIRE].

[30] M. Gorbahn and U. Haisch, Indirect probes of the trilinear Higgs coupling: $g g \rightarrow h$ and $h \rightarrow \gamma \gamma$, JHEP 10 (2016) 094 [arXiv: 1607.03773] [INSPIRE].

[31] W. Bizon, M. Gorbahn, U. Haisch and G. Zanderighi, Constraints on the trilinear Higgs coupling from vector boson fusion and associated Higgs production at the LHC, arXiv: 1610.05771 [INSPIRE].

[32] A. Sirlin, Role of $\sin ^{2} \theta_{W}\left(m_{Z}\right)$ at the $Z^{0}$ peak, Phys. Lett. B 232 (1989) 123 [INSPIRE]. 
[33] S. Fanchiotti and A. Sirlin, Accurate determination of $\sin ^{2} \theta_{W}\left(m_{z}\right)$, Phys. Rev. D 41 (1990) 319 [INSPIRE].

[34] G. Degrassi, S. Fanchiotti and A. Sirlin, Relations between the on-shell and MS frameworks and the $M(W)-M(Z)$ interdependence, Nucl. Phys. B 351 (1991) 49 [inSPIRE].

[35] G. Degrassi, P. Gambino and P.P. Giardino, The $m_{W}-m_{Z}$ interdependence in the standard model: a new scrutiny, JHEP 05 (2015) 154 [arXiv:1411.7040] [INSPIRE].

[36] P. Gambino and A. Sirlin, Relation between $\sin ^{2} \theta_{W}\left(m_{z}\right)$ and $\sin ^{2} \theta_{\text {eff }}^{\text {lep }}$ Phys. Rev. D 49 (1994) 1160 [hep-ph/9309326] [INSPIRE].

[37] A. Sirlin, Radiative corrections in the $\mathrm{SU}(2)_{L} \times \mathrm{U}(1)$ theory: a simple renormalization framework, Phys. Rev. D 22 (1980) 971 [INSPIRE].

[38] T. Hahn, Generating Feynman diagrams and amplitudes with FeynArts 3, Comput. Phys. Commun. 140 (2001) 418 [hep-ph/0012260] [INSPIRE].

[39] R. Mertig, M. Böhm and A. Denner, FEYN CALC: computer algebraic calculation of Feynman amplitudes, Comput. Phys. Commun. 64 (1991) 345 [INSPIRE].

[40] V. Shtabovenko, R. Mertig and F. Orellana, New developments in FeynCalc 9.0, Comput. Phys. Commun. 207 (2016) 432 [arXiv:1601.01167] [INSPIRE].

[41] R. Mertig and R. Scharf, TARCER: a Mathematica program for the reduction of two loop propagator integrals, Comput. Phys. Commun. 111 (1998) 265 [hep-ph/9801383] [INSPIRE].

[42] A.I. Davydychev and J.B. Tausk, Two loop selfenergy diagrams with different masses and the momentum expansion, Nucl. Phys. B 397 (1993) 123 [INSPIRE].

[43] S.P. Martin, Evaluation of two loop selfenergy basis integrals using differential equations, Phys. Rev. D 68 (2003) 075002 [hep-ph/0307101] [INSPIRE].

[44] S.P. Martin and D.G. Robertson, TSIL: a program for the calculation of two-loop self-energy integrals, Comput. Phys. Commun. 174 (2006) 133 [hep-ph/0501132] [INSPIRE].

[45] A. Sirlin and R. Zucchini, Dependence of the quartic coupling $H(m)$ on $M(h)$ and the possible onset of new physics in the Higgs sector of the standard model, Nucl. Phys. B 266 (1986) 389 [INSPIRE].

[46] ATLAS collaboration, Measurement of the $W$-boson mass in pp collisions at $\sqrt{s}=7 \mathrm{TeV}$ with the ATLAS detector, arXiv:1701.07240 [INSPIRE].

[47] Particle Data Group collaboration, C. Patrignani et al., Review of particle physics, Chin. Phys. C 40 (2016) 100001 [InSPIRE].

[48] CDF collaboration, T.A. Aaltonen et al., Measurement of $\sin ^{2} \theta_{\mathrm{eff}}^{\text {lept }}$ using $e^{+} e^{-}$pairs from $\gamma^{*} / Z$ bosons produced in $p \bar{p}$ collisions at a center-of-momentum energy of $1.96 \mathrm{TeV}$, Phys. Rev. D 93 (2016) 112016 [arXiv:1605.02719] [InSPIRE].

[49] D0 collaboration, V.M. Abazov et al., Measurement of the effective weak mixing angle in $p \bar{p} \rightarrow Z / \gamma^{*} \rightarrow e^{+} e^{-}$events, Phys. Rev. Lett. 115 (2015) 041801 [arXiv:1408.5016] [INSPIRE].

[50] M. Awramik, M. Czakon and A. Freitas, Bosonic corrections to the effective weak mixing angle at $O\left(\alpha^{2}\right)$, Phys. Lett. B 642 (2006) 563 [hep-ph/0605339] [INSPIRE].

[51] C. Grojean, G. Servant and J.D. Wells, First-order electroweak phase transition in the standard model with a low cutoff, Phys. Rev. D 71 (2005) 036001 [hep-ph/0407019] [INSPIRE]. 
[52] P. Huang, A. Joglekar, B. Li and C.E.M. Wagner, Probing the electroweak phase transition at the LHC, Phys. Rev. D 93 (2016) 055049 [arXiv: 1512.00068] [INSPIRE].

[53] J.M. Cornwall, D.N. Levin and G. Tiktopoulos, Derivation of gauge invariance from high-energy unitarity bounds on the s matrix, Phys. Rev. D 10 (1974) 1145 [Erratum ibid. D 11 (1975) 972] [INSPIRE].

[54] A. Falkowski, Three slides on triple Higgs couplings, talk given at the LHC Higgs Cross Section Working Group, HH subgroup meeting, December 12, Geneva, Switzerland (2016). 